\title{
The clinical and cellular basis of contact lens-related corneal infections
}

\author{
Danielle M Robertson \\ H Dwight Cavanagh
}

Department of Ophthalmology, The University of Texas Southwestern Medical Center, Dallas, TX, USA
Correspondence: Danielle M Robertson Department of Ophthalmology, UT Southwestern Medical Center, 5323 Harry Hines Blvd, Dallas, TX 75390-9057, USA

Tel + I 2146483416

Fax +I 2146488447

Email danielle.robertson@

utsouthwestern.edu

\begin{abstract}
Microbial keratitis (MK) is the most visually devastating complication associated with contact lens wear. Pseudomonas aeruginosa (PA) is highly invasive in the corneal epithelium and is responsible for more than half of the reported cases of contact lens-related MK. To protect against Pseudomonas-mediated MK, the corneal epithelium has evolved overlapping defense mechanisms that function to protect the ocular surface from microbial invasion. Research has shown that contact lens wear disrupts these protective mechanisms through breakdown of normal homeostatic surface renewal as well as damaging the corneal surface, exposing underlying cell membrane receptors that bind and internalize PA through the formation of lipid rafts. Human clinical trials have shown that initial adherence of PA with resulting increased risk for microbial infection is mediated in part by contact lens oxygen transmissibility. Recently, chemical preserved multipurpose solutions (MPS) have been implicated in increasing PA adherence to corneal epithelial cells, in addition to inducing significant levels of toxic staining when used in conjunction with specific silicone hydrogel lenses. This review summarizes what is currently known about the relationship between contact lenses, the corneal epithelium, MPS, and infection.
\end{abstract}

Keywords: cornea, epithelium, contact lens, microbial keratitis

\section{Introduction}

The corneal epithelium is a multi-layered stratified epithelial sheet that undergoes continuous exposure to potential invasive ocular pathogens. Despite these recurrent attacks, the epithelium maintains a high level of resistance against microbial invasion, which is critical to preventing infection and preserving vision. This arsenal of defense includes the antimicrobial properties of the tear film and self-defense capabilities of the epithelium itself, including the desquamation of apoptotic cells from the surface of the cornea, tight cellular junctions comprising the epithelial barrier, and the production of ocular surface mucins by apical cells which contribute to the innermost surface of the tear film as well as acting to reduce bacterial adherence. Breakdown of these collective mechanisms predispose the cornea to infection and are enhanced by penetration through the basal lamina.

Clinically, the overall result of these cellular events is the presence of a light-blocking infiltrate with an overlying epithelial defect. Notably, trauma, pre-existing ocular surface disease, and contact lens wear have been earmarked as the most common etiologies of microbial infection, with Pseudomonas aeruginosa (PA) identified as the primary causative organism (Ormerod and Smith 1986; Pachigolla et al 2007; Mondino et al 1986). Research directed at understanding and reducing PA-mediated contact lens microbial keratitis has steadily progressed over the past several decades. Hallmark epidemiological studies have established for the first time the relative risk of infection with contact lenses, particularly with extended wear, and patient associated risk factors have been identified (Schein et al 1989; Poggio et al 1989; Keay et al 2006; Morgan et al 2005). 
Lens-induced hypoxia was recognized as a key mediator for microbial invasion with PA and newer lens designs and types followed with increased oxygen transmissibility in hopes of improving lens safety (Holden and Mertz 1984).

Likewise, contact lens care solutions have evolved from the use of homemade saline to chemically preserved no-rub multipurpose solutions which currently account for a significant portion of the lens care market. With the efficacy of "no-rub" cleaning recently being called into question however; and, the current influx of increased reports of contact-lens related microbial infections in the news media, the effectiveness of these lens care solutions and their components as adequate antimicrobial disinfectants is being re-evaluated. In addition, the combination of these chemically preserved products with specific silicone hydrogel lens polymers has raised a new issue relating to the joint biocompatibility of these materials with the ocular surface. The purpose of this review is to summarize the current body of research relating to contact lenses, the corneal epithelium, multipurpose lens care solutions, and infection for basic scientists and practicing clinicians.

\section{Corneal epithelial physiology and contact lens wear}

The corneal epithelium is a self-renewing, stratified epithelial sheet that provides the first-line of defense against microorganisms invading the eye and a smooth refractive surface essential for vision. Contact lenses share an intimate relationship with the epithelial surface; and all forms of contact lens wear, regardless of lens material and modality of wear, have a profound effect on the physiology of this tissue. For more than two decades, researchers have focused on elucidating the effects of contact lens wear on the epithelium in both clinical and laboratory based studies in hopes of identifying a potential linkage between these events and the risk for contact lens-related infection. Importantly, the substantial cumulation of these studies have shown that the physical presence of a contact lens, irrespective of oxygen transmissibility, disrupts corneal epithelial renewal mechanisms, producing a thinned, stagnant epithelium; however, the impact of this stagnation as it relates to infection is unknown (Ladage et al 2002; Holden et al 1985).

It is well established that the continual supply of epithelial cells originates in the limbus, where limbal stem cells, the gatekeepers of epithelial renewal reside. Offspring from the limbus migrate centrally, wherein basal epithelial cells undergo a single round of cell division, with both daughter cells migrating vertically toward the ocular surface (Ren et al 1999). Preliminary studies on the effects of contact lens wear on central epithelial proliferation in the rabbit demonstrated for the first time that contact lenses inhibited mitosis in the basal layer of the epithelium (Hamano et al 1983). Later studies using BrdU, a marker for proliferating cells, confirmed that contact lens wear results in a reduction in proliferation in basal cells through out the central cornea and the degree of mitotic suppression was regulated in part by lens oxygen transmission (Figure 1A) (Ren et al 1999; Ladage et al 2003; Ladage et al 2001). Similarly, triple-labeling studies using BrdU, the cell cycle marker Ki67, and propidium iodide in the rabbit cornea have further shown that not only is proliferation reduced, but contact lens wear results in a concurrent decrease in the vertical migration of terminally differentiated cells as they migrate out of the basal cell layer toward the corneal surface (Ladage et al 2003). This delay in differentiation and renewal is accompanied by an observable increase in surface epithelial cell size, presumably due to the retention of older cells on the surface of the epithelium (Ladage et al 2002; Lemp et al 1990; Mathers et al 1992; Ren et al 1999).

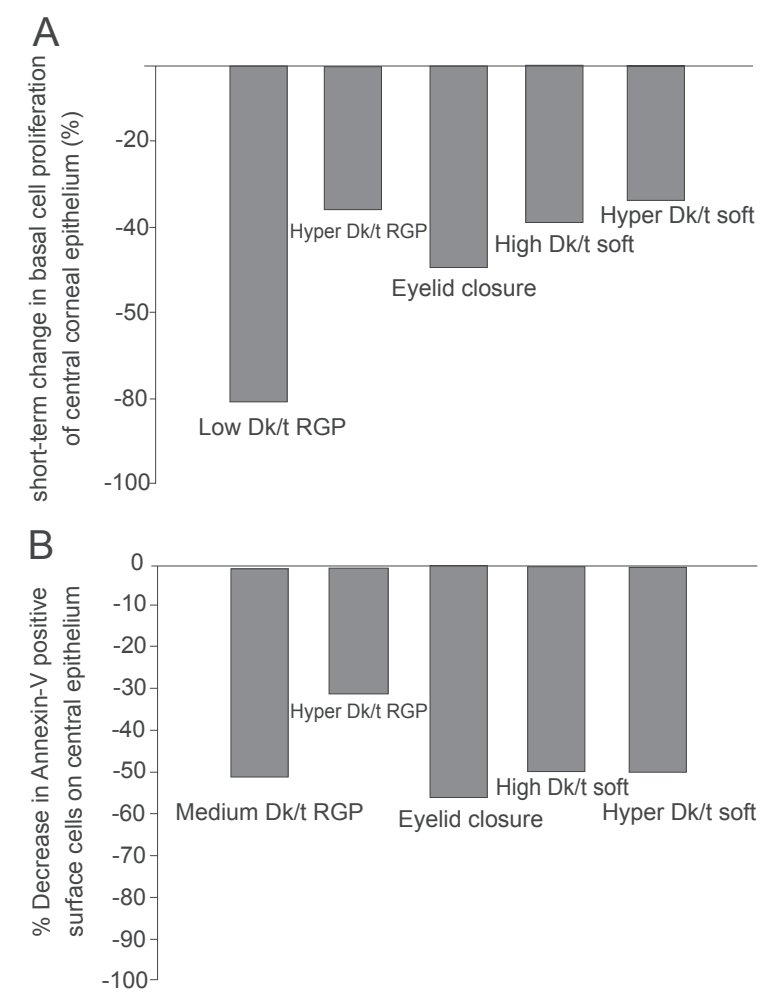

Figure I (A) The effect of contact lens wear on basal cell proliferation. Single pulse BrdU labeling in the rabbit corneal epithelium following 24 hours of contact lens wear demonstrated a reduction in basal cells mitosis in all lens groups tested and from hypoxia induced by eyelid suturing, suggestive of both lens- and oxygen-mediated effects. (B) The effect of contact lens wear on apoptosis in the central corneal epithelium. Annexin $\mathrm{V}$ labeling, an early marker for apoptosis, also showed a reduction in epithelial desquamation with all forms of contact lens wear, which did not appear to be related to lens-oxygen transmissibility. Copyright (c) 2008. Figures adapted with permission from Ladage PM, Yamamoto K, Li L, et al. 2002. Corneal epithelial homeostasis following daily and overnight lens wear. Contact Lens Anterior Eye, 25: I I-21. 
This retention of older cells on the surface of the epithelium is a result of an inhibition in apoptosis-driven surface cell desquamation into the preocular tear film (Ren and Wilson 1996). In the noncontact lens wearing eye, the majority of epithelial cells desquamate from the central corneal ( $\mathrm{Li}$ et al 2002; Yamamoto et al 2002). Mechanical forces from the eyelid during blinking may be responsible for this unique geographic pattern, likely through activation of stress proteins along the epithelial surface. In addition, the ability of epithelial cells to continually shed in a controlled manner represent an important component in the innate immune response, as cells infected with PA initiate apoptosis and shed from the surface of the normal eye, providing inherent anti-infective protection. In contrast, in the contact lens wearing eye, central epithelial desquamation is markedly reduced (Ren et al 1999; Ladage et al 2001). Whole mount studies in the rabbit cornea using Annexin V and Ethidium labeling following 24 hours of contact lens wear have further demonstrated a significant reduction in central epithelial cell death for both rigid gas permeable (RGP) and soft contact lenses with varying levels of oxygen transmissibility, as well as prolonged eyelid closure (Figure 1B) (Li et al 2002; Yamamoto et al 2002). These findings have been further related to changes in the pattern of epithelial cell localization of the antiapoptotic protein BCL2. In both rabbit and human tissue, BCL2 localizes to the nuclei of surface central corneal epithelial cells and disappears or becomes undetectable prior to positive TUNEL labeling, indicating apoptosis has ensued (Yamamoto et al 2001). In the contact lens wearing eye, co-localization experiments with BCL2 and TUNEL following 24 hours of contact lens wear reveals persistent nuclear BCL2 expression in central surface cells corresponding to a loss of positive TUNEL labeling (Yamamoto et al 2001). Collectively, these findings indicate severe reduction of normal epithelial desquamation at the cellular level secondary to daily or overnight lens wear, which is insensitive to lens oxygen transmission levels. When challenged with microbial invasion, the inhibition of the apoptotic response also represents a further breakdown in the barrier function of the cornea.

\section{Corneal epithelial damage, bacterial adherence and internalization}

Contact lenses can also produce varying levels of direct corneal epithelial surface damage, further compromising the tight cell to cell barrier function. Critical early animal studies in the rabbit model using both rigid and hydrogel contact lenses demonstrated that oxygen played an important role in contact lens-related cell loss and damage to the underlying corneal epithelium. In these early studies, lactate dehyrdrogenase (LDH), an enzyme that is released by damaged or dying cells, was used as a measure of the direct effects of contact-lens hypoxia and lens pressure on the epithelial surface (Ichijima et al 1992). Specifically, analysis of the effects of seven-day extended wear of high $(\mathrm{Dk} / \mathrm{t}=34)$, super high $(\mathrm{Dk} / \mathrm{t}=56)$, and ultra high $(\mathrm{Dk} / \mathrm{t}=64)$ oxygen transmissible RGP contact lenses demonstrated that both high and super high lens wear significantly increased LDH activity, whereas ultra high oxygen transmitting lenses failed to increase LDH activity compared to controls (Imayasu et al 1993). Additional studies using scanning electron microscopy and tandem scanning confocal microscopy confirmed that polymethylmethacrylate (PMMA) lenses with zero oxygen transmissibility induced robust epithelial surface damage, whereas RGP lenses with varying levels of oxygen transmissibility induced milder levels of surface cell loss (Imayasu et al 1994). Significantly, the rigid lens with the highest oxygen transmissibility $(\mathrm{Dk} / \mathrm{t}=64)$ resulting in an equivalent oxygen percentage of 19.13 produced no apparent epithelial surface damage. In comparison, all soft hydrogel lenses induced more moderate levels of surface cell loss at all lens oxygen levels studied (Imayasu et al 1994).

These surface damage findings were further correlated with PA adherence to the cornea following 24 hours of lens wear, demonstrating that PMMA and RGP lenses with low oxygen transmissibility all significantly increased PA bound to the corneal surface; likewise, the three hydrogel lenses tested significantly bound PA as well (Imayasu et al 1994). This increase in bacterial adhesion for rigid and soft lenses suggested that there was increased PA receptor exposure on deeper epithelial cells following loss of the protective outer layer. Specifically, these studies provided the first direct experimental data illustrating that the lower the oxygen transmissibility of the lens, the greater the degree of ocular surface damage $(\mathrm{R}=0.993, \mathrm{P}<0.01)$ and the greater the amount of PA adhered to the corneal surface $(\mathrm{R}=0.998, \mathrm{P}<0.01)$, regardless of lens rigidity (Figure 2) (Imayasu et al 1994). However, when rigidity was accounted for and RGP lenses were compared to soft hydrogel lenses at the same lens oxygen transmissibility levels, rigid lenses induced more surface damage but less PA binding than their soft lens counterparts. Notably, these findings are further consistent with a similar study in rabbits using a direct, injury induced wound healing model. In this parallel study, the authors used filter paper to create a partial thickness injury to remove the superficial epithelial cell layer as well as denuded epithelium with stromal exposure. In all eyes examined, there was an increase in PA 
binding to deeper cell layers following partial injury compared to undisturbed eyes, with the greatest increase in bacterial adherence seen at the stromal surface in the denuded cornea; as the epithelial defect resolved over time, PA adherence returned to baseline values (Klotz et al 1989).

In the corneal epithelium, PA has been shown not only to bind to the plasma membrane, but also to possess the novel ability to invade internally into intact epithelial cells (Fleiszig et al 1994). Recent studies investigating the mechanism of PA binding and internalization in corneal epithelial cells have established an entry route for bacteria to the cell interior through fluid structures in the cell membrane known as lipid rafts (Yamamoto et al 2006; Yamamoto et al 2005). Lipid rafts are cholesterol and glycosphingolipid enriched micro-domains which function as signaling platforms to mediate a host of cellular signaling functions, including microbial internalization (Simons and Ikonen 1997; Shin and Abraham 2001; Shin and Abraham 2001; Duncan et al 2002). Once inside the cell,

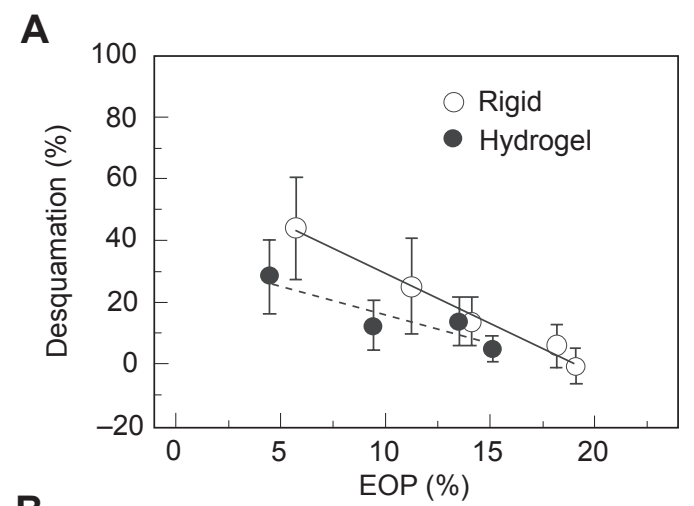

B

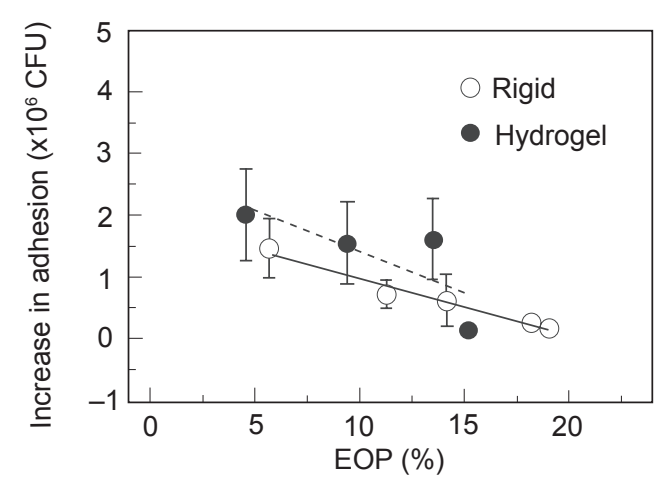

Figure 2 (A) Percentage of epithelial desquamation as a function of equivalent oxygen percentage (EOP). As lens oxygen transmissibility increased, there was a significant decrease in epithelial desquamation for both RGP and hydrogel lenses. At lower oxygen transmission levels, RGP lenses induced greater levels of surface epithelial desquamation. At the highest EOP tested, RGP lenses induced no observable desquamation. (B) Increase in PA adherence, expressed as colony forming units (CFU), as a function of EOP. Similar to desquamation, increasing EOP levels resulted in a reduction of PA adherence. In contrast to desquamation, for each level of oxygen transmission, rigid lenses bound significantly less PA than hydrogel lenses. Copyright (C) 1994. Figures adapted with permission from Imayasu M, Petroll WM, Jester JV, et al. 1994.The relation between contact lens oxygen transmissibility and binding of Pseudomonas aeruginosa to the cornea after overnight wear. Ophthalmology, 101:371-88. the normal host response to microbial invasion is to initiate apoptosis and shedding of the infected cell from the surface of eye (Grassme and Kirschnek 2000). In contact lens wear however, the mechanism regulating epithelial apoptosis has been altered, allowing PA to replicate within the cellular cytoplasm and providing a safe reservoir from commonly used extracellular aminoglycoside antibiotics.

In the rabbit eye, 24 hours of PMMA lens wear induced lipid raft formation in the central and peripheral epithelium (Figure 3) (Yamamoto et al 2006). Following lens removal and subsequent incubation in PA, lipid raft expressing cells preferentially bound PA compared to nonraft expressing cells. Laser confocal microscopy confirmed the direct association of PA with the lipid raft fraction of the cell as well as the intracellular localization of the bacteria. In contrast, despite uniform localization of B-cholera toxin, a marker for lipid rafts, in the limbal and conjunctival epithelium, no PA adherence was detected. To dissect out the mechanical effects of the contact lens from hypoxia on raft formation, eyelid suturing experiments were performed to induce chronic hypoxia in the absence of a contact lens (Yamamoto et al 2006). While these suturing experiments
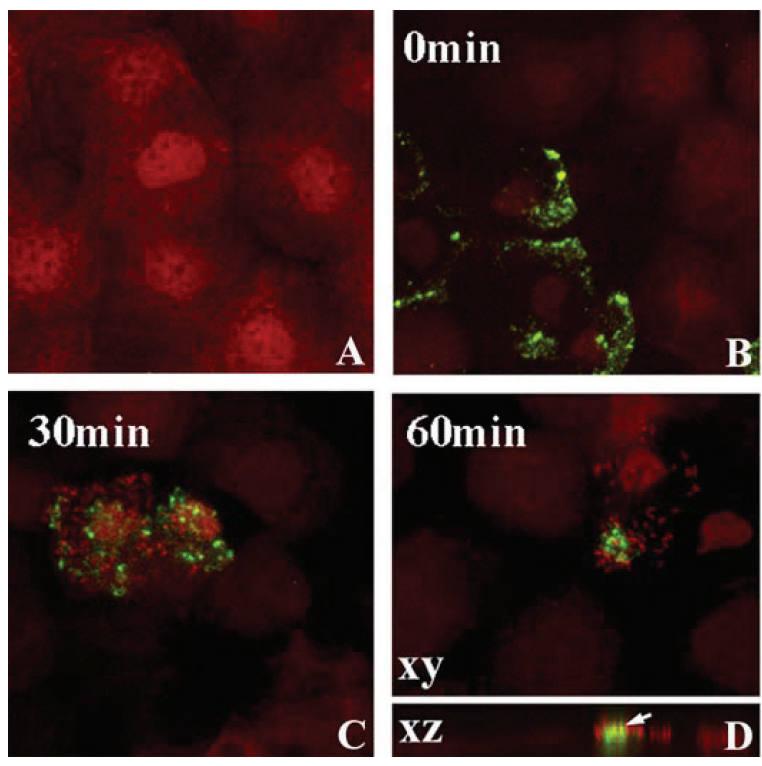

Figure 3 Lipid raft expression in the rabbit corneal epithelium. After 24 hours of PMMA lens wear, whole mount tissue was labeled with beta cholera toxin (green) and counterstained with PI (red) to label epithelial nuclei and allow for visualization of PA. (A) Undisturbed corneal epithelium. No lipid raft expression was seen in the absence of a contact lens. (B) After 24 hours of lens wear, pre-infection with PA, lipid rafts were detected in surface epithelial cells, evident by green punctate staining. (C)Thirty minutes after infection with PA, PA were seen to preferentially adhere to lipid raft expressing cells. (D)At I hour post infection, PA appeared to cluster around the lipid raft fraction of the plasma membrane. In the vertical XZ plane, lipid rafts appeared to associate directly with the lipid raft and internalization was noted. Copyright (C) 2005. Image adapted with permission from Yamamoto N, Yamamoto N, PetrollWM, et al. 2005. Internalization of Pseudomonas aeruginosa is mediated by lipid rafts in contact lens-wearing rabbit and cultured human corneal epithelial cells. Invest Ophthalmol Vis Sci, 46:1348-55. 
demonstrated an upregulation of lipid raft expression in the central cornea, there was no detectable increase in intracellular PA, as measured by Gentamicin survival assays. In contrast, 3 days of low Dk RGP extended lens wear upregulated lipid rafts in the peripheral rabbit epithelium (outside the central $6 \mathrm{~mm}$ ) with subsequent PA adherence and internalization (Yamamoto et al 2006). Interestingly, this peripheral raft localization corresponds to a commonly reported geographic region associated with corneal infiltrative events in response to silicone hydrogel lens wear (Schein et al 2005).

Cystic fibrosis transmembrane conductance regulator (CFTR), a chloride anion channel, has been proposed to be the epithelial receptor responsible for binding PA and mediating internalization through lipid rafts (Kowalski and Pier 2004). In corneal epithelial cells cultured in the presence of serum, hypoxia has been shown to regulate CFTR expression and subsequent bacterial internalization (Zaidi et al 2004). More recently, CFTR has also been shown to recruit major vault protein (MVP) which appears to play an essential role in mediating the subsequent immune response in pulmonary epithelial cells (Kowalski et al 2007). In contrast to these studies, in vitro cell culture experiments using a unique telomerase immortalized corneal epithelial cell line grown in the absence of serum failed to establish a functional role for CFTR in lipid raft internalization (Yamamoto et al 2006; Robertson et al 2005). The results from these serum-free studies correlated with in vivo contact lens animal studies which confirmed the presence of CFTR in the corneal epithelium; however, such studies failed to detect a role for CFTR in PA internalization. Significantly, CFTR did not co-localize with lipid raft expressing cells and blocking peptides targeting amino acids 108-117 and 103-117, the functional site on the CFTR receptor, failed to alter PA internalization. Collectively, these findings suggest that the functional activation of the CFTR receptor in epithelial cells may be regulated by the differential effects of serum or cell type and that disparate mechanisms may be responsible for the internalization of PA in ocular trauma where the epithelium is serum-exposed, compared to the contact-lens wearing model where the post-lens tear film is a serum-free environment.

\section{Bacterial binding: An effective marker?}

In order for in vitro findings on changes in epithelial apoptosis and bacterial adherence to be clinically relevant, it is imperative to establish in vivo markers that can be tested and applied in a clinical setting. Based upon the well accepted view that the initial inciting step in infection is PA binding to the corneal epithelium, a technique to evaluate bacterial binding to exfoliated corneal epithelial cells ex vivo was proposed (Fullard and Wison 1986; Fleiszig et al 1992; Ren et al 2007). This noninvasive irrigation technique uses sterile saline to gently remove exfoliated cells from the corneal surface for subsequent incubation in a well-established invasive strain of PA (strain 27853, American Type Culture Collection, Rockville, MD) (Figure 4A). Promising initial reports implementing this technique in human subjects reported an increase in PA adherence to exfoliated corneal epithelial cells following extended hydrogel lens wear (Fleiszig et al 1992). In an effort to justify the use of this technique in large scale human clinical studies, the adherence of PA to exfoliated cells from the rabbit corneal surface was compared to the adherence of PA to the residual rabbit corneal surface following irrigation (Ren et al 1997). In this study, rabbits were fit with rigid gas permeable or soft contact lenses stratified by oxygen transmissibility. After 24 hours, exfoliated cells were collected and both exfoliated cells and the remaining corneal surface were incubated in PA. After 30 minutes of bacterial exposure, the number of PA bound to the total corneal surface following contact lens wear correlated with $\mathrm{PA}$ adherence to shed corneal cells $(\mathrm{R}=0.78, \mathrm{P}<0.001$, Figure 4B). Significantly, this finding validated this ex vivo method as an indirect measure of bacterial binding in vivo (Ren et al 1997).

Implementing this methodology, the role of the contact lens and hypoxia was evaluated in a series of successive, prospective human clinical trials using RGP, hydrogel, and silicone hydrogel lens designs and identical study protocols. In the initial pilot study, 109 patients wore lenses on a six-night extended wear schedule over three months and exfoliation rates as well as PA adherence were measured after 24 hours of overnight and 3 months of extended wear (Ren et al 1999). The significant outcome for this study was that lens material did not regulate PA adherence; however, the lens oxygen transmissibility was inversely correlated with PA adherence to exfoliated cells. In addition, the use of hyper Dk lens materials failed to increase PA adherence compared to controls. In another study, 246 patients wore either soft or RGP lenses on a daily wear basis for 4 weeks (Ladage et al 2001). In comparison to the pilot, both high and hyper oxygen transmissible soft lenses significantly upregulated PA binding, with high oxygen transmissible lenses binding much higher levels of PA than their hyper oxygen transmissible counterparts. Interestingly, hyper Dk RGP lenses did not affect PA adherence; however, 
all test lenses significantly reduced epithelial desquamation ( $\mathrm{P}<0.001)$ (Ladage et al 2001).

The long term effects of extended contact lens wear on the corneal epithelium were then investigated in 178 patients in 6 or 30 night extended wear over one year (Ren et al 2002). Similar to the previous results, this study again showed that while hyper oxygen transmissible lenses had a significant increase in PA adherence, this increase was considerably lower than PA adherence following high Dk lens wear; and again, hyper Dk RGP lenses failed to upregulate PA adherence (Ren et al 2002). Additionally, there were two interesting and unexpected findings in this study: there was no significant difference between 6 and 30 night extended wear of the hyper oxygen lens materials and there appeared to be an adaptive physiological recovery to PA adherence at 9 and 12 months of extended wear for all lenses tested (Ren et al 2002). A subsequent clinical trial evaluating 136 patients over 1 year of extended lens wear further confirmed these findings (Cavanagh et al 2002).

The collective results from these clinical studies indicate that the combined presence of a lens and reduced availability of oxygen are critical factors in mediating bacterial binding. From these findings, relative risk of infection was established and the predicted safety of contact lens wear correlated exactly with previous historical epidemiological data allowing for stratification based upon lens type and wearing modality (RGP DW/EW < soft DW < soft EW lenses) (Schein et al 1989; Poggio et al 1989; Ren et al 2002). Additionally, these findings also demonstrated an unexpected adaptive return to baseline values, predicting for the first time that the risk of microbial keratitis was highest during the first six months of lens wear; a subsequent epidemiological study has recently confirmed this prediction (Stapleton et al 2008).

A third key finding illustrated by these clinical studies was the repeated observation that hyper oxygen transmissible hydrogel lenses bound significantly less PA than high oxygen transmissible lens wear. Not surprisingly, this finding lent further support to a role for oxygen transmission in the regulation of PA binding, calling for an in vivo human study to delineate the role of oxygen from the mechanical effects of the lens. In order to accomplish this, 10 subjects were fitted with goggles that exposed the corneal surface to conditions of hypoxia and anoxia for up to 6 hours of wear (Ren et al 1999). Significantly, while hypoxia down-regulated epithelial desquamation similar to contact lens wear, there was no corresponding increase in PA adherence to shed cells, establishing for the first time that hypoxia alone does not regulate $\mathrm{PA}$ adherence, but the presence of a lens is required (Ren et al 1999).
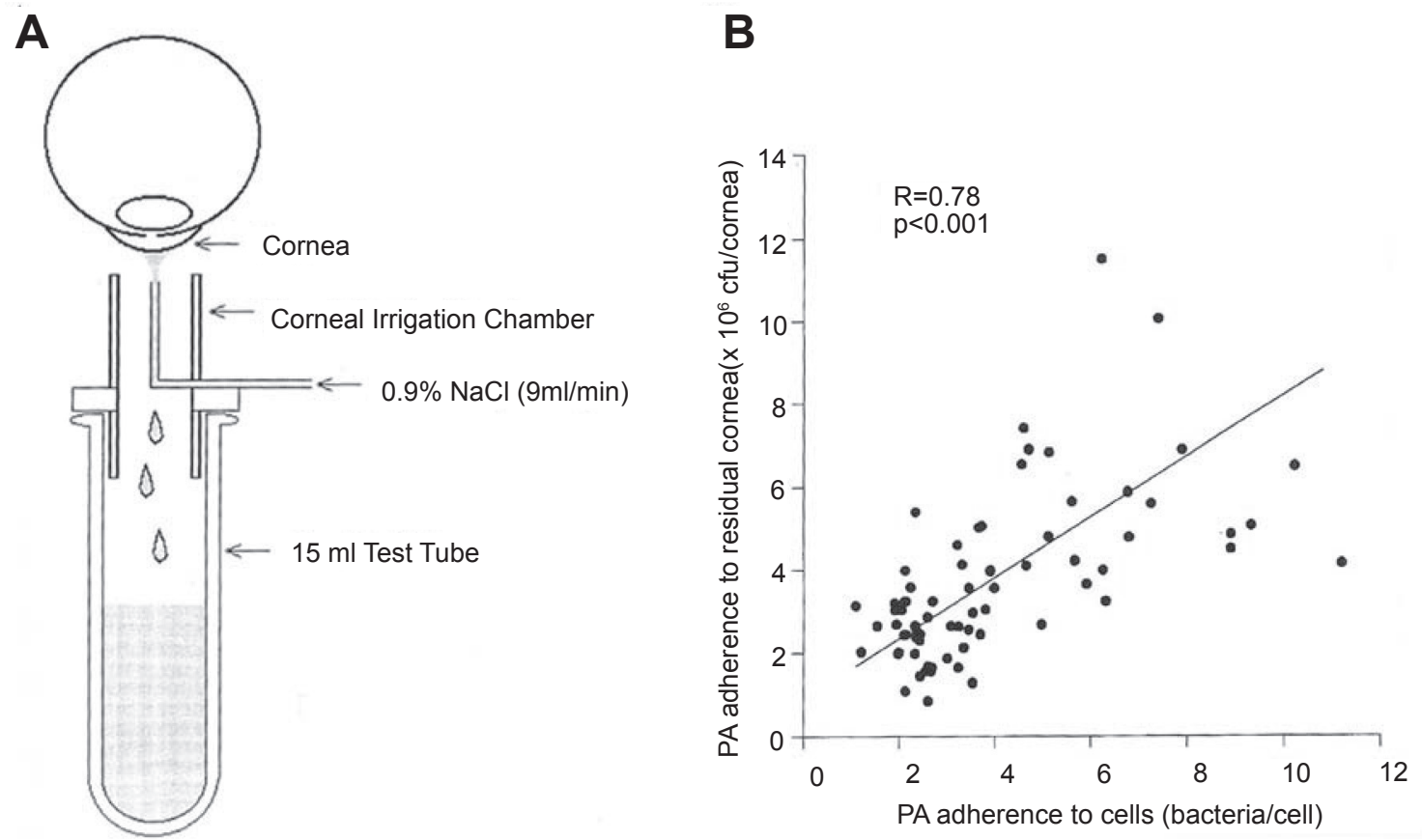

Figure 4 (A) Schematic of the noninvasive ocular irrigation device. Warmed saline is gently applied to the eye and collected in a test tube directly below. Cells are then incubated in an invasive strain of PA for 30 minutes, vacuum filtered onto polycarbonate filters, and stained with acridine orange for visualization using an epifluorescent microscope. (B) Correlation of PA adherence between exfoliated corneal epithelial cells and the residual corneal surface $(\mathrm{R}=0.78, \mathrm{P}<0.00 \mathrm{I})$. Copyright $@ \mathrm{I}$ I997. Images adapted with permission from Ren DH, Petroll WM, Jester JV, et al. 1997. Adherence of Pseudomonas aeruginosa to shed rabbit corneal epithelial cells after overnight wear of contact lenses. CLAO J, 23:63-8. 
While hypoxia may have a corroborative role in PA adherence, a disparity in PA adherence between the 3 month pilot study and the remaining clinical trials exists. Specifically, in patients wearing silicone hydrogel lenses, there was no increase seen in the initial pilot (Ren et al 1999), compared to significant increases in PA adherence as early as 24 hours following lens wear in the latter studies (Ladage et al 2001; Ren et al 2002; Cavanagh et al 2002). In evaluating this data, it is important to note that the initial three month study utilized a nonpreserved contact lens care solution. Thus it appears that the absence of an increase in PA binding seen with hyper oxygen transmissible soft lenses may be due to the use of a nonpreserved care solution compared to the increase in PA adherence seen with the same lens using chemically preserved MPS (Ren et al 1999). To further examine the effect of preserved solutions on PA adherence, the most recent clinical trial evaluated 121 silicone hydrogel lens wearers using hydrogen-peroxide based solutions in both daily and 6/30 night extended wear over one year. In agreement with the 3 month data, the use of a nonpreserved hydrogen peroxide-based solution in this study eliminated the increase in PA binding that was seen with MPS usage (Ren et al 1999; Robertson et al 2008). Irrespective of differences in PA binding, corneal epithelial homeostasis was still altered, with decreases seen in epithelial desquamation and central epithelial thickness similar to that of previously reported studies; no adaptive effects of either daily or extended wear were seen.

Significantly, the finding that preservatives in MPS solutions may play a role in altering epithelial desquamation and PA adherence is not novel and has been previously reported in a clinical study investigating the use of chemical preservatives on the cornea in the absence of a contact lens (Figure 5) (Li et al 2003). In this prospective cross-over clinical trial, patients instilled one of four solutions into the eye 6 times a day for four days. For all solutions tested, the instillation of MPS resulted in a significant decrease in exfoliation of shed corneal epithelial cells with a corresponding significant increase in PA binding. The results from this study coincide with an even earlier study evaluating the effects of contact lenses and care solutions on the rabbit corneal epithelium, which found that care solutions using hydrogen peroxide demonstrated 3 times greater PA attachment than PBS, whereas preserved solutions using Dymed or Polyquad increased PA adherence by 8 and 24 times, respectively (Williams et al 1990). Taken together, while a potential underlying mechanism responsible for these increases in PA adherence is unknown, these data highlight the possibility that the use of chemically preserved MPS may interact in conjunction with contact lens wear to increase PA adherence to the corneal epithelium synergistically; and therefore may further increase the risk for contact lens-related microbial infection.

\section{Lens-care solutions, corneal staining and adverse events}

The potential role of contact lens care solutions in microbial keratitis has recently gained significant interest due to increased reports of fungal and Acanthamoeba keratitis in the news media. In addition, since the widespread acceptance of silicone hydrogel lenses into the marketplace, there have also been a significant number of reports of increased solutioninduced corneal staining associated with specific silicone hydrogel contact lens/solution combinations (Andrasko and Ryen 2007; Jones et al 2002; Garofalo et al 2005). This increase in solution-induced corneal staining is of interest, as the use of silicone hydrogel lenses has not reduced the

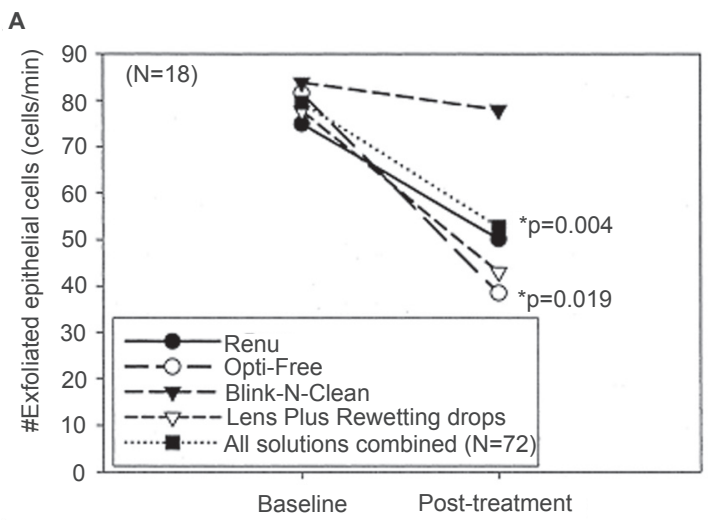

B

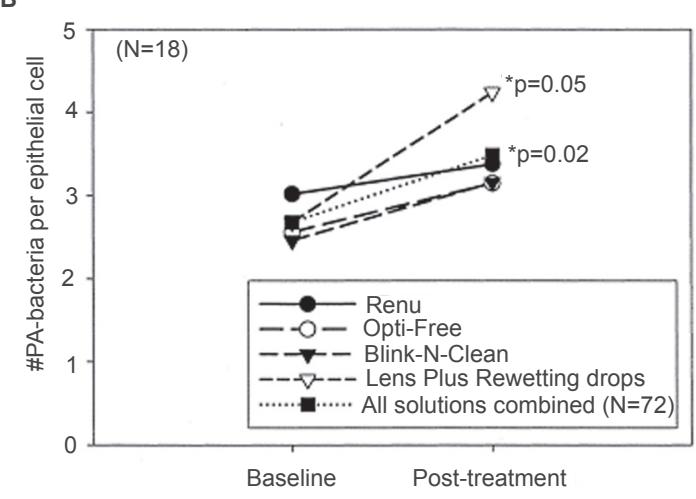

Figure 5 (A) The number of exfoliated cells (cells/min) before and after treatment. Surface cell exfoliation was reduced after repeated exposure to chemically preserved MPS. In addition, Lens Plus which contains the buffering agent boric acid, also showed a significant effect on the decrease in the number of cells shed from the epithelial surface. (B) The number of PA bound per epithelial cell before and after treatment. Concurrent with a decrease in desquamation, there was an increase in PA adherence seen with all solutions tested. Copyright (C) 2003. Figures adapted with permission from Li SL, Ladage PM,Yamamoto T, et al. 2003. Effects of contact lens care solutions on surface exfoliation and bacterial binding to corneal epithelial cells. Eye Contact Lens, 29:27-30. 
rates of $\mathrm{MK}$ as expected. In contrast to silicone hydrogel lens wear, RGP lenses, which carry the lowest risk for infection, can induce significant amounts of 3 and 9 o'clock staining in the limbal region. Significantly, when compared to the recent studies on lipid raft internalization, the inability of functional lipid rafts to form in the limbal epithelium in response to lens wear suggests that it is the location of fluorescein staining within the corneal epithelium that relates to the potential risk for infection and not the presence of staining alone.

More recent reports support the view that corneal staining may be directly related to inflammation. Retrospective clinical studies have shown that contact lens solutioninduced corneal staining is associated with a 3 times greater risk of corneal infiltrative events with a positive correlation between the two (Carnt et al 2007). Similar to clinical trials, this report also found that the least amount of toxic staining was seen with the use of hydrogen peroxide care solutions (Carnt et al 2007; Cho et al 1998). Of note, this latter finding also corresponds to the low amount of toxic staining seen with hydrogen peroxide care solutions compared to multipurpose solutions previously reported elsewhere.

At the cellular level, early studies using the in vivo confocal microscope investigated the effect of different contact lens care solutions coupled with a single hydrogel lens and demonstrated differential effects on the corneal epithelium. Interestingly, these confocal studies showed effects on the corneal epithelium long before biomicroscopy findings were evident, suggesting that staining alone is not a sensitive measure of epithelial disruption. This study also demonstrated that when used with the same hydrogel contact lens, different preservatives and buffer systems had different effects on the corneal epithelium (Chang et al 1999). In support of this view, more recent studies reported significant cytotoxic effects in vitro based upon inhibition of cell growth from commonly used MPS as well as the various agents used in their formulations (Mowrey-Mckee et al 2002; Santodomingo-Rubido et al 2006).

In terms of barrier function, numerous attempts have been made to examine and clarify the effects of contact lens solutions on the epithelial surface. Many of these studies have focused on the localization of the tight junction associated protein ZO-1; whereas others have focused on measurements of the epithelial barrier function, using both fluorescein permeability assays as well as measurements of transepithelial resistance (Imayasu et al 2008; Bernal and Ubels 1991). In a recent study, the use of four commonly used MPS on corneal epithelial cell monolayer culture showed a breakdown of ZO-1, along with a decrease in transepithelial resistance, indicating a disruption in the epithelial barrier (Imayasu et al 2008). It is likely that the reported breakdown of ZO-1 is the in vitro corollary to solution induced toxic staining seen at the corneal surface; however, restrain must still be exercised when extrapolating serum-exposed monolayer toxicity culture data to increased rates of $\mathrm{MK}$ in vivo.

\section{Discussion}

Pseudomonas aeruginosa is a pathogenic Gram-negative microbe that is highly infectious in the corneal epithelium and is the most common pathogen associated with contact lens wear (Ormerod and Smith 1986; Mondino et al 1986). In the undisturbed eye, the corneal epithelium utilizes a host of defense mechanisms to deflect invasion by PA; however, following contact lens wear, these innate defense mechanisms are collectively compromised. This compromise includes the inhibition of apoptotic desquamation and a slowed renewal mechanism, producing a thinned, stagnant epithelial sheet (Ladage et al 2002). The relationship between contact lens wear and alterations in epithelial homeostasis as well as the implications of delayed renewal in the pathogenesis of MK are unknown. While it is likely that the alteration or loss of one of these defense mechanisms may place the host at an increased risk for infection, it is well understood that the loss of one mechanism alone is not sufficient to induce infection in animal models. This resilient property of the epithelium is due to the incredible redundancy built into this biological system. Thus, it appears that it is the cumulative breakdown of these collective processes that results in contact lens related $\mathrm{MK}$ and further illustrates the multifactorial nature of the disease process.

A significant amount of research has shown that contact lenses damage the corneal epithelium, with the greatest amount of damage being seen with low oxygen transmissible RGP lenses (Imayasu et al 1994). From the results of our clinical trials however, it is now known that at similar oxygen transmissibility levels, even though rigid lenses induce substantially more epithelial surface damage than hydrogel lenses, corneal epithelial cells inherently bind more PA after hydrogel lens wear than their rigid counterparts (Ren et al 1999; Ladage et al 2001; Ren et al 2002). The overall results from these studies suggest two possible conclusions; (1) reduced tear flow and stagnation in the post lens tear film of hydrogel lens wearers may increase PA binding to surface corneal epithelial cells by trapping or increasing bacterial exposure to the ocular surface as opposed to wear of RGP lenses which permit adequate tear flushing; (2) while damage 
to the epithelial surface disrupts the tight barrier function of the corneal epithelium and exposes receptors on underlying squamous cells, PA binding is not solely related to epithelial damage alone.

In addition to lens-induced corneal damage, chemically preserved MPS have been shown to induce a transient "toxic" staining event at the corneal surface (Carnt et al 2007). MPS toxic staining has been shown to be a risk factor for corneal inflammatory events, however, thus far there has been no correlation between staining and $\mathrm{MK}$ (Carnt et al 2007). Importantly, while no association has been established, the use of chemically preserved MPS in human clinical trials has been shown to reduce epithelial desquamation in concert with an upregulation of PA-binding receptors in surface epithelial cells in both the presence and absence of a contact lens ( $\mathrm{Li}$ et al 2003). This increase in PA binding to corneal epithelial cells is a well-established risk factor for infection. Therefore, it may be that the use of these chemical preservatives and buffering agents in contact lens solutions may be altering cell surface protein expression in corneal epithelial cells and upregulating PA receptor expression independent of the clinical manifestation seen as staining.

Solution-induced staining aside, in addition to increased PA adherence, contact lens wear and MPS alike inhibit epithelial desquamation ( $\mathrm{Li}$ et al 2003). The inability of an epithelial cell to desquamate following invasion results in a failure of the body to rid itself of the infecting organism. This mechanism of internalization has been established to occur through glycosphingolipid- and cholesterol-enriched raft formation in the plasma membrane (Yamamoto et al 2005). Uniquely, contact lens-induced corneal lipid raft formation and preferential PA adherence to raft-expressing cells has been localized to the central and peripheral epithelium, corresponding to the pattern of localization of MK (Yamamoto et al 2006). In contrast, limbal and conjunctival epithelium which uniformly label with beta cholera toxin fail to form functional raft areas and do not exhibit any detectable level of PA adherence or internalization. These findings suggest that raft formation and bacterial internalization within the ocular surface epithelia is restricted to the central and peripheral corneal epithelium, corresponding to the established clinical phenotypes seen in epidemiological studies (Schein et al 2005).

Further studies are necessary to understand the mechanism by which contact lenses induce lipid raft formation in the corneal epithelium and receptor expression facilitating PA adherence. CFTR has been proposed as one potential receptor; however, there are conflicting reports on the role of CFTR in PA internalization (Yamamoto et al 2006; Kowalski and Pier 2004). This disparity arises from the use of serum in culture media and represents an important aspect of in vitro cell culture experimentation, as corneal cells in vivo are maintained in a relatively serum free environment in the nonwounded cornea. Thus, further studies are needed to examine the differential effects of serum on receptor expression in vitro with direct comparison to events in the in vivo animal eye.

In order to produce PA infection in the cornea in animal models, a break in the basal lamina is required, leading to stromal invasion. Invasive PA strains internalize into epithelial cells, while other cytotoxic strains of PA migrate between cells to the basal surface where they exert their destructive effect through the injection of cytotoxins into the cell interior (Fleiszig et al 1996; Fleiszig et al 1997). The combined intracellular and paracellular migration results in the localization of PA beneath the epithelial sheet in apposition to the basal lamina (Fleiszig 2006). Presumably factors secreted by either the epithelial cells in response to cellular damage and bacteria or the bacteria themselves lead to the eventual destruction and penetration of the basal lamina. While breakdown of the various layers of the epithelial defense mechanism along the way each represent a potential increased risk factor for infection, a cumulative series of events appears to be required in order for an MK to ensue.

Given the multifactorial nature of the disease process, it may be some time before we truly understand the mechanisms underlying contact-lens related $\mathrm{MK}$ and establish safe, therapeutic measures to prevent it. The collective findings summarized in this review however, provide a useful strategy for maximizing safe contact lens wear based upon the current lens materials and care solutions available. RGP lenses made with hyper oxygen transmissible lens materials by far provide the safest option for patients electing to undergo daily or extended lens wear. While soft contact lenses inherently carry a higher risk, likely due to the stagnation or cesspool that is created in the post lens tear film, the use of hyper oxygen transmissible silicone hydrogel lens materials coupled with nonpreserved hydrogen peroxide care solutions can minimize the risk for microbial keratitis.

\section{Disclosure}

Supported in Part by NIH Grant K08 EY15713 (DMR), EY10738 (HDC), Infrastructure Grant EY016664, The Pearle Vision Foundation, Dallas, Texas, and an unrestricted 
grant from Research to Prevent Blindness, Inc., New York, New York. The authors report no conflicts of interest in this work.

\section{References}

Andrasko G and Ryen K. 2007. A series of evaluations of MPS and silicone hydrogel lens combinations. Review of Cornea and Contact Lenses, March:36-42.

Bernal DL and Ubels JL. 1991. Quantitative evaluation of the corneal epithelial barrier: Effect of artificial tears and preservatives. Curr Eye Res, 10:645-56.

Carnt N, Jalbert I, Stretton S, et al. 2007. Solution toxicity in soft contact lens daily wear is associated with corneal inflammation. Optom Vis Sci, 84:309-15.

Cavanagh HD, Ladage PM, Li SL, et al. 2002. Effects of daily and overnight wear of a novel hyper oxygen-transmissible soft contact lens on bacterial binding and corneal epithelium. Ophthalmology, 109:1957-69.

Chang JH, Ren H, Petroll WM, et al. The application of in vivo confocal microscopy and tear LDH measurement in assessing corneal response to contact lens and contact lens solutions. Curr Eye Res, 1999;19:171-81.

Cho P, Lui T, Kee CS. 1998. Soft contact lens care systems and corneal staining in Hong Kong-Chinese. Contact Lens Anterior Eye, 21:47-53.

Duncan MJ, Shin JS, Abraham SN, et al. 2002. Microbial entry through caveolae: variations on a theme. Cell Microbiol, 4:783-91.

Fleiszig SMJ, Efron N, Pier GB, et al. 1992. Extended contact lens wear enhances Pseudomonas aerugniosa adherence to human corneal epithelium. Invest Ophthalmol Vis Sci, 33:2908-16.

Fleiszig SMJ, Zaidi TS, Preston MJ, et al. 1996. Relationship between cytotoxicity and corneal epithelial cell invasion by clinical isolates of Pseudomonas aeruginosa. Infect Immun, 64:2288-94.

Fleiszig SMJ, Evans DJ, Do N, et al. 1997. Epithelial cell polarity affects susceptibility to Pseudmonas aerugnisa invasion and cytotoxicity. Infect Immun, 65:2861-7.

Fleiszig SMJ, Zaidi T, Fletcher EL, et al. 1994. Pseudomonas aeruginosa invades corneal epithelial cells during experimental Infect Immun, 62:3485-93.

Fleiszig SMJ. 2006. The pathogenesis of contact lens-related keratitis. Optom Vis Sci, 83:E866-E73.

Fullard RJ and Wison GS. 1986. Investigation of sloughed corneal epithelial cells collected by non-invasive irrigation of the corneal surface. Curr Eye Res, 5:847-56.

Garofalo RJ, Dassanayake N, Carey C, et al. 2005. Corneal staining and subjective symptoms with multipurpose solutions as a function of time. Eye Contact Lens, 31:166-74.

Grassme H and Kirschnek S. 2000. CD95/CD95 ligand interactions on epithelial cells in host defense to Pseudomonas aeruginosa. Science, 290:527-30.

Hamano H, Hori M, Hamano T, et al. 1983. Effects of contact lens wear on mitosis of corneal epithelium and lactate content in aqueous humor of rabbit. Jpn J Ophthalmol, 27:451-8.

Holden BA and Mertz GW. 1984. Critical oxygen levels to avoid corneal edema for daily and extended wear contact lenses. Invest Ophthalmol Vis Sci, 25:1161-7.

Holden BA, Sweeney DF, Vannas A, et al. 1985. Effects of long-term extended contact lens wear on the human cornea. Invest Ophthalmol Vis Sci, 26:1489-501.

Ichijima H, Imayasu M, Ohashi J, et al. 1992. Tear lactate dehydrogenase levels: a new method to assess effects of contact lens wear in man. Cornea, 11:114-20.

Imayasu M, Moriyama T, Ohashi J, et al. 1993. Effects of rigid gas permeable contact lens extended wear on rabbit cornea assessed by LDH activity, MDH activitiy, and albumin levels in tear fluid. CLAO J, 19:153-7.

Imayasu M, Petroll WM, Jester JV, et al. 1994. The relation between contact lens oxygen transmissibility and binding of Pseudomonas aeruginosa to the cornea after overnight wear. Ophthalmology, 101:371-88.
Imayasu M, Shiraishi A, Ohashi Y, et al. 2008. Effects of multipurpose solutions on corneal epithelial tight junctions. Eye Contact Lens, 34:50-5.

Jones L, MacDougall N, Sorbara LG, et al. 2002. Asymptomatic corneal staining associated with the use of balafilcon silicone-hydrogel contact lenses disinfected with a polyaminopropyl biguanide-preserved care regimen. Optom Vis Sci, 79:753-61.

Keay L, Edwards K, Naduvilath T, et al. 2006. Microbial keratitis: predisposing factors and morbidity. Ophthalmology, 113:109-16.

Klotz SA, Au YK, Misra RP, et al. 1989. A partial-thickness epithelial defect increases the adherence of Pseudomonas aeruginosa to the cornea. Invest Ophthalmol Vis Sci, 30:1069-74.

Kowalski MP and Pier GB. 2004. Localization of cystic fibrosis transmembrane conductance regulator to lipid rafts of epithelial cells is required for Pseudomonas aeruginosa-induced cellular activation. J Immunol, $172: 418-25$.

Kowalski MP, Dubouix-Bourandy A, Bajmoczi M, et al. 2007. Host resistance to lung infection mediated by major vault protein in epithelial cells. Science, 317:130-2.

Ladage PM, Yamamoto K, Ren DH, et al. 2001. Effects of rigid and soft contact lens daily wear on corneal epithelium, tear lactate dehydrogenase, and bacterial binding to exfoliated epithelial cells. Ophthalmology, 108:1279-88.

Ladage PM, Yamamoto K, Ren DH, et al. 2001. Proliferation rate of rabbit corneal epithelium during overnight rigid contact lens wear. Invest Ophthalmol Vis Sci, 42:2804-12.

Ladage PM, Yamamoto K, Li L, et al. 2002. Corneal epithelial homeostasis following daily and overnight lens wear. Contact Lens Anterior Eye, 25:11-21.

Ladage PM, Jester JV, Petroll WM, et al. 2003. Vertical movement of epithelial basal cells toward the corneal surface during use of extended-wear contact lenses. Invest Ophthalmol Vis Sci, 44:1056-63.

Ladage PM, Ren DH, Petroll WM, et al. 2003. Effects of eyelid closure and disposable and silicone hydrogel extended contact lens wear on rabbit corneal epithelial cell proliferation. Invest Ophthalmol Vis Sci, 44:1843-9.

Lemp MA, Mathers WD, Sachdev MS, et al. 1990. The effects of contact lens wear on the morphology of corneal surface cells in the human. Trans Am Ophthalmol Soc, 88:313-25.

Li L, Ren DH, Ladage PM, et al. 2002. Annexin V binding to rabbit corneal epithelial cells following overnight contact lens wear or eyelid closure. CLAO J, 28:48-54.

Li SL, Ladage PM, Yamamoto T, et al. 2003. Effects of contact lens care solutions on surface exfoliation and bacterial binding to corneal epithelial cells. Eye Contact Lens, 29:27-30.

Mathers WD, Sachdev MS, Petroll WM, et al. 1992. Morphologic effects of contact lens wear on the corneal surface. CLAO J, 18:49-52.

Mondino BJ, Weissman BA, Farb MD, et al. 1986. Corneal ulcers associated with daily-wear and extended-wear contact lenses. Am J Ophthalmol, 102: 58-65.

Morgan PB, Efron N, Brennan NA, et al. 2005. Risk factors for the development of corneal infiltrative events associated with contact lens wear. Invest Ophthalmol Vis Sci, 46:3136-43.

Mowrey-Mckee M, Sills A, Wright A, et al. 2002. Comparative cytotoxcity potential of soft contact lens care regimens. CLAO J, 28:160-4.

Ormerod LD and Smith RE. 1986. Contact lens-associated microbial keratitis. Arch Ophthalmol, 104:79-83.

Pachigolla G, Blomquist P, Cavanagh HD, et al. 2007. Microbial keratitis pathogens and antibiotic susceptibilities: a 5-year review of cases at an urban county hospital in North Texas. Eye Contact Lens, 33:45-9.

Poggio EC, Glynn RJ, Schein OD, et al. 1989. The incidence of ulcerative keratitis among users of daily-wear and extended-wear soft contact lenses. N Engl J Med, 321:779-83.

Ren DH, Petroll WM, Jester JV, et al. 1999. Short-term hypoxia downregulates epithelial cell desquamation in vivo, but does not increase Pseudomonas aeruginosa adherence to exfoliated human corneal epithelial cells. CLAO J, 25:73-9. 
Ren DH, Petroll WM, Jester JV, et al. 1999. The effect of rigid gas permeable contact lens wear on proliferation of rabbit corneal and conjunctival epithelial cells. CLAO J, 25:136-41.

Ren DH, Petroll WM, Jester JV, et al. 1999. The Relationship between contact lens oxygen permeability and binding of Pseudomonas aeruginosa to human corneal epithelial cells after overnight and extended Wear. CLAO J, 25:80-100.

Ren DH, Petroll WM, Jester JV, et al. 1997. Adherence of Pseudomonas aeruginosa to shed rabbit corneal epithelial cells after overnight wear of contact lenses. CLAO J, 23:63-8.

Ren DH, Yamamoto K, Ladage PM, et al. 2002. Adaptive effects of 30-night wear of hyper-O2 transmissible contact lenses on bacterial binding and corneal epithelium. Ophthalmology, 109:27-39.

Ren H and Wilson G. 1996. Apoptosis in the corneal epithelium. Invest Ophthalmol Vis Sci, 37:1017-25.

Robertson DM, Li L, Fisher S, et al. 2005. Characterization of growth and differentiation in a telomerase-immortalized human corneal epithelial cell line. Invest Ophthalmol Vis Sci, 46:470-8.

Robertson DM, Petroll WM, Cavanagh HD, et al. 2008. The effect of nonpreserved care solutions on 12 months of daily and extended silicone hydrogel contact lens wear. Invest Ophthalmol Vis Sci, 49:7-15.

Santodomingo-Rubido J, Mori O, Kawaminami S, et al. 2006. Cytotoxicity and antimicrobial activity of six multipurpose soft contact lens disinfecting solutions. Ophthal Physiol Opt, 26:476-82.

Schein OD, Glynn RJ, Poggio EC, et al. 1989. The relative risk of ulcerative keratitis among users of daily-wear and extended-wear soft contact lenses. A case-control study. Microbial Keratitis Study Group. NEngl J Med, 321:773-8

Schein OD, McNally JJ, Katz J, et al. 2005. The incidence of microbial keratitis among wearers of a 30-day silicone hydrogel extended-wear contact lens. Ophthalmology, 112:2172-9.

Shin JS and Abraham SN. 2001 Caveolae as portals of entry for microbes. Microbes Infect, 3:755-61.
Shin JS and Abraham SN. 2001. Co-option of endocytic functions of cellular caveolae by pathogens. Immunology, 102:2-7.

Simons K and Ikonen E. 1997. Functional rafts in cell membranes. Nature, 387:569-72.

Stapleton F, Keay L, Edwards K, et al. 2008. The incidence of contact lens related microbial keratitis in Australia. Ophthalmology, Jun 4. [Epub ahead of print].

Williams WF, Totaro ME, Currie JP, et al. 1990. Investigation of the effects of contact lens wear and various lens care products on adherence of Pseudomonas aeruginosa. Invest Ophthalmol Vis Sci, 31(Suppl):550.

Yamamoto K, Ladage PM, Ren DH, et al. 2001. Bcl-2 expression in the human cornea. Exp Eye Res, 73:247-55.

Yamamoto K, Ladage PM, Ren DH, et al. 2001. Effects of low and hyper Dk rigid gas permeable contact lenses on $\mathrm{Bcl}-2$ expression and apoptosis in the rabbit corneal epithelium. CLAO J, 27:137-43.

Yamamoto K, Ladage PM, Ren DH, et al. 2002. Effect of eyelid closure and overnight contact lens wear on viability of surface epithelial cells in rabbit cornea. Cornea, 21:85-90.

Yamamoto N, Yamamoto N, Petroll WM, et al. 2005. Internalization of Pseudomonas aeruginosa is mediated by lipid rafts in contact lens-wearing rabbit and cultured human corneal epithelial cells. Invest Ophthalmol Vis Sci, 46:1348-55.

Yamamoto N, Yamamoto N, Jester JV, et al. 2006. Prolonged hypoxia induces lipid raft formation and increases Pseudomonas internalization in vivo after contact lens wear and lid closure. Eye Contact Lens, 32:114-20.

Yamamoto N, Yamamoto N, Petroll WM, et al. 2006. Regulation of Pseudomonas aeruginosa internalization after contact lens wear in vivo and in serum-free culture by ocular surface cells. Invest Ophthalmol Vis Sci, 47:3430-40.

Zaidi T, Mowrey-Mckee M, Pier GB, et al. 2004. Hypoxia increases corneal cell expression of CFTR leading to increased Psuedomonas aeruginosa binding, internalization, and initiation of inflammation. Invest Ophthalmol Vis Sci, 45:4066-74. 
